\title{
Avoiding the effect of $B C G$ vaccination in detecting Mycobacterium tuberculosis infection with a blood test
}

\author{
R. Diel*, M. Ernst" ${ }^{\#}$, G. Döscher", L. Visuri-Karbe+, U. Greinert", S. Niemann ${ }^{\S}$, \\ A. Nienhaus ${ }^{f}$ and C. Lange
}

ABSTRACT: Bacille Calmette-Guérin (BCG) vaccination can confound tuberculin skin test (TST) reactions in the diagnosis of latent tuberculosis infection (LTBI).

The TST was compared with a Mycobacterium tuberculosis (MTB)-specific enzyme-linked immunospot (ELISPOT) assay during an outbreak of MTB infection at a police academy in Germany.

Participants were grouped according to their risk of LTBI in close $(n=36)$ or occasional $(n=333)$ contacts to the index case. For the TST, the positive response rate was $53 \%$ (19 out of 36) among close and 16\% (52 out of 333) among occasional contacts. In total, 56 TST-positive contacts (56 out of $71=78.9 \%$ ) and 27 TST-negative controls (27 out of $298=9.1 \%$ ) underwent ELISPOT testing. The odds ratio (OR) of a positive test result across the two groups was 29.2 (95\% confidence interval (Cl) 3.5-245.0) for the ELISPOT and 19.7 (95\% Cl 2.0-190.2) for the TST with a $5 \mathrm{~mm}$ cutoff. Of 369 contacts, $158(42.8 \%)$ had previously received BCG vaccination. The overall agreement between the TST and the ELISPOT was low, and positive TST reactions were confounded by BCG vaccination (OR $4.8(95 \% \mathrm{Cl} 1.3-18.0)$ ). In contrast, use of a 10-mm induration cut-off for the TST among occasional contacts showed strong agreement between TST and ELISPOT in nonvaccinated persons.

In bacille Calmette-Guérin-vaccinated individuals, the Mycobacterium tuberculosis-specific enzyme-linked immunospot assay is a better indicator for the risk of latent tuberculosis infection than the tuberculin skin test.

KEYWORDS: Contact tracing, early secretory antigenic target 6, enzyme-linked immunospot assay, interferon- $\gamma$, latent tuberculosis infection, tuberculosis

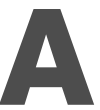
pproximately one-third of mankind is currently infected with Mycobacterium tuberculosis (MTB), causing significant morbidity and mortality with $>2$ million deaths $\cdot \mathrm{yr}^{-1}$ [1]. Tuberculosis (TB) control programmes aim to decrease the incidence of TB by interruption of the transmission of MTB. Contact tracing and treatment of individuals with latent $\mathrm{TB}$ infection (LTBI) are the key components of TB control programmes. In addition, other important key components of TB control are the improvement of case finding among persons presenting with symptoms of TB, especially in HIV-infected individuals, by providing better access to quality-assured TB sputum microscopy as well as the Directly Observed Therapy Short-Course strategy in TB treatment. The latter achieves higher cure rates by an uninterrupted supply of antituberculotic drugs

For editorial comments see page 1. in a standardised short-course chemotherapy under direct observation [2].

By applying classical epidemiological and molecular strain-typing techniques, population-based studies have recently revealed a high frequency of transmission of MTB, even in countries with a low TB incidence [3-7]. The tuberculin skin test (TST) introduced by Mantoux has been widely used as a screening test to identify individuals with LTBI. In screening for LTBI, the predictive value of a positive TST result (positive predictive value (PPV)) is the probability that a contact with a positive TST result is truly infected and, consequently, may benefit from chemotherapy [8]. This single parameter combines, on the one hand, the sensitivity and specificity of the method and, on the other, the prevalence of TB infection within the population subset examined. In close contacts, the prevalence of infection plays only a minor role in determining the PPV,
AFFILIATIONS

${ }^{*}$ School of Public Health, University of Düsseldorf, Düsseldorf, \#Division of Immune Cell Analysis, -Division of Clinical Infectious Diseases,

${ }^{\S}$ Division of Mycobacteriology, Research Center Borstel, Borstel, ${ }^{+}$Medical Service, Police Academy Schleswig-Holstein, Eutin, and fInstitution for Statutory Accident Insurance and Prevention in the Health and Welfare Services, Hamburg, Germany.

\section{CORRESPONDENCE}

C. Lange

Medical Clinic

Research Center Borstel

Parkallee 35

23845 Borstel

Germany

Fax: 494537188313

E-mail: clange@fz-borstel.de

Received:

September 122005

Accepted after revision:

February 072006 
because their risk of MTB transmission exceeds by far the risk in the general population. Thus, after this step of contact investigation, the PPV is primarily influenced by the specificity of the test method: the higher the specificity (i.e. a low level of false-positive results), the higher the PPV of a positive result.

One problem in the clinical applicability of the Mantoux test is its cross-reactivity with antigens present in other mycobacteria, such as the $M$. bovis bacillus Calmette-Guérin (BCG) vaccine strain and environmental mycobacterial species. This crossreactivity leads to false-positive results and decreases the PPV of the TST. Therefore, alternative diagnostic tools for the detection of LTBI have been explored. One antigen of MTB that induces a strong interferon (IFN)- $\gamma$-secreting CD4 T-cell immune response in experimental models $[9,10]$, and that is absent in $M$. bovis BCG and most other nontuberculous mycobacteria, is the early secretory antigenic target 6 (ESAT6). Counting of ESAT-6-reactive peripheral blood mononuclear cells (PBMC) by detection of IFN- $\gamma$-spot-forming cells (enzyme-linked immunospot (ELISPOT) assay) has been used successfully to identify persons with active and latent MTB infection [11-16].

Whereas the sensitivity of an MTB-specific ELISPOT assay appears comparable to that of the TST [17], the specificity of this test may actually be superior to that of the classical TST. However, studies that addressed this question have so far either been conducted in a country with intermediate MTB incidence [18] (as opposed to the low MTB incidence encountered in most European countries and the USA) or with children [19]. Until now, the ELISPOT assay has not been evaluated for adult contacts directly related to a single source case in a low incidence setting.

From January 2004, extensive contact tracing became necessary in young and middle-aged adults within a police academy in Eutin, a town in the German federal state of SchleswigHolstein. In mid-September 2003, one of its cadets (a 31-yr-old unmarried former military policeman, who had been BCGvaccinated in 1973) complained of progressive cough and weakness. After a temporary improvement of his condition on broad-spectrum antibiotic therapy, the patient experienced haemoptysis in December 2003. A chest radiograph at that time showed extensive infiltrates and cavitations in the right upper lobe, and he was admitted to a pulmonary clinic. Sputum samples showed 3+ acid-fast bacilli on Ziehl-Neelsen stain. Isolates were culturally confirmed as MTB, susceptible to firstline drugs. Despite standard quadruple antituberculous therapy acid-fast bacilli were still present on sputum smears and MTB could still be cultured from the sputum up to 2 months after treatment had been initiated. Thus, the patient had to be considered as having been an index patient, highly infectious to contact persons at the barracks of the police academy, presumably for a period of up to 4 months.

The present authors participated in contact tracing at the police academy barracks, and compared the agreement between TST and an ESAT-6-based ELISPOT for the identification of LTBI.

\section{MATERIALS AND METHODS Study population}

A contact investigation at the police academy yielded a total of 369 persons with identified risk of LTBI due to exposure to the index case since September 2003. Of these, 287 were born in the former West Germany, 74 (20.1\%) in the former East Germany and eight persons were foreign-born and had migrated to Germany.

The mean (range) age of the contacts was 28 (15-62) yrs. Contacts were predominantly male $(n=265 ; 71.8 \%)$. No subject reported any contact with persons with TB before exposure to the index case. Thirty-six persons $(9.8 \%)$ were considered to be close contacts (fig. 1); these comprised 35 police cadets, who were sharing the same classroom and sleeping quarters with the index case (first-year groups 5 and 6) and one of the instructors (see below) who had trained the index case in cardiorespiratory resuscitation.

The other 333 contacts had only occasional exposure to the index case during his presumed period of infectiousness, and did not vary substantially with respect to exposure time or proximity. These contacts comprised 187 further cadets, 46 instructors and 100 individuals working as members of the vehicle maintenance staff, administration, kitchen personnel, cleaners or uniform store-keepers (fig. 1).

As required by the current national guidelines for contact tracing in Germany [20] (issued by the DZK (German Central Committee for the Control of TB)) all these contacts were subjected to initial Mantoux TST testing 12 weeks following their last possible exposure to the index case. The TST was performed on the volar aspect of the forearm, and results were read 48-72 $\mathrm{h}$ later. Chest radiographs of the TST-positive contacts were performed, and clinical and radiographic findings were reviewed for evidence of active TB. None of the contacts reported that they were seropositive for HIV, undergoing haemodialysis, currently being treated with corticosteroids or other immunosuppressives, or known to have a malignant disease or diabetes mellitus. None of the nonvaccinated contacts had previously been involved in contact tracing or had given a positive result in an employment-related investigation.

Of all the 369 contacts, 158 (42.8\%) had a history of previous BCG vaccination; for this reason, and because the efficacy of LTBI treatment is highly dependent on the proportion of true MTB infections, the skin test-positive persons were offered the possibility of being tested by ELISPOT in order to provide more information about the PPV of the Mantoux test. For practical reasons, only every tenth TST-negative individual was selected for ELISPOT testing by randomisation to provide a control group. All individuals agreed to participate in the study.

\section{Cell cultures}

PBMC were prepared by Ficoll-Hypaque density-gradient centrifugation from heparinised blood and put into culture in RPMI 1640 medium containing $100 \mathrm{U} \cdot \mathrm{mL}^{-1}$ penicillin, $100 \mu \mathrm{g} \cdot \mathrm{mL}^{-1}$ streptomycin, $2 \mathrm{mmol} \cdot \mathrm{L}^{-1}$ L-glutamine and $5 \%$ foetal calf serum (culture medium).

\section{ELISPOT assay}

ELISPOT assays for human IFN- $\gamma$ were performed according to the manufacturer's guidelines (Autoimmun Diagnostika (AID), Strasberg, Germany). Briefly, 200,000 PBMC were plated overnight on 96-well plates, which had been pre-coated with a mouse anti-human IFN- $\gamma$ antibody, in $200 \mu \mathrm{L}$ culture 


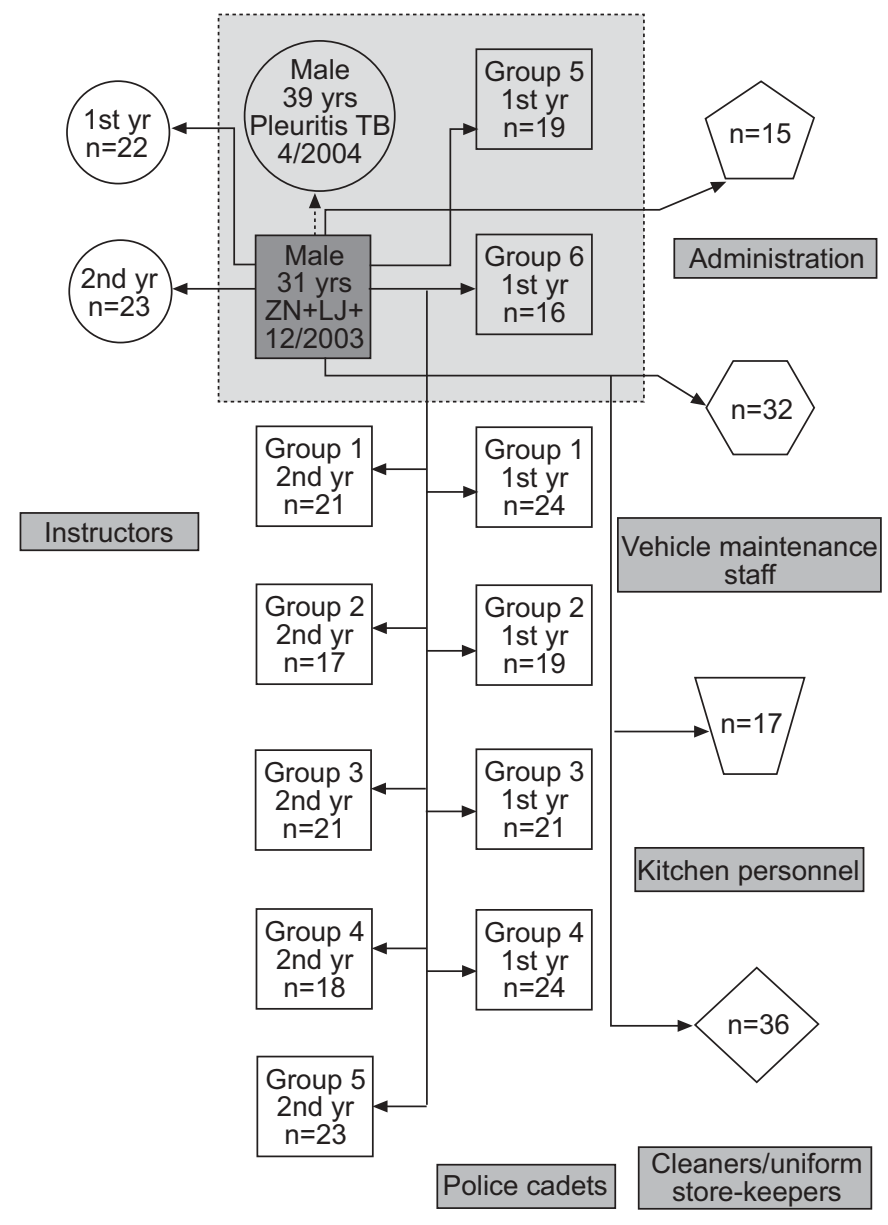

FIGURE 1. Pattern of exposure among 369 contacts of a police cadet with $3+$ acid-fast bacillus smear-positive pulmonary tuberculosis (TB). ZN: Ziehl-Neelsen; LJ: Loewenstein-Jensen medium. 1 : close contactcs; 1 : source case; black arrow: prospectively registered contacts.

medium per well. The cells were left unstimulated (negative control), were stimulated with $10 \mathrm{ng} \cdot \mathrm{mL}^{-1}$ anti-CD3 monoclonal antibody (clone X35; Beckman-Coulter, Krefeld, Germany; positive control), with $10 \mu \mathrm{g} \cdot \mathrm{mL}^{-1}$ of purified protein derivative of tuberculin (PPD; Statens Serum Institute, Copenhagen, Denmark) and with $5 \mu \mathrm{g} \cdot \mathrm{mL}^{-1}$ of ESAT- 6 antigens (kindly provided by AID). After $20 \mathrm{~h}$ of culture at $37^{\circ} \mathrm{C}$, the plates were washed and then incubated at room temperature for $2.5 \mathrm{~h}$ in the presence of a biotinylated mouse antihuman IFN- $\gamma$ monoclonal antibody. The plates were again washed repeatedly and then, after a 2-h incubation with streptavidin-conjugated horseradish peroxidase, were washed again and the substrate buffer (containing 3-amino-9ethylcarbazole and $\mathrm{H}_{2} \mathrm{O}_{2}$ ) was added. The reaction was stopped after 20-30 min by the addition of water. After repeated washing with water, the plates were allowed to dry. Visualisation and analysis of the spots was performed by using the AID EliSpot Reader System ELR02 (AID). The response of stimulated cultures was considered positive if the test well contained at least five more spots than the negative control well, and this number was at least twice that in negative control wells. The background number of spots in negative control wells was always $<5$ spots per well.

\section{Spoligotype pattern Spacer

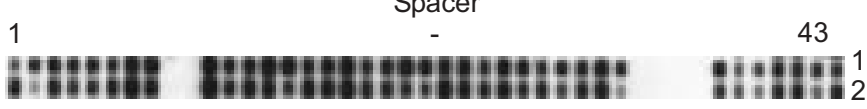

FIGURE 2. Spoligotyping patterns of Mycobacterium tuberculosis isolates of the index case and a contact who developed pleural tuberculosis.

\section{Statistical analysis}

Categorical data were compared using the Chi-squared test (or Fisher's exact test, when expected cell sizes were smaller than five). Concordance between TST and ELISPOT assay results was assessed using $\kappa$ coefficients. Values $<40 \%$ indicate weak correlation (if any); values of $0.41-0.60$ indicate good agreement and values $>0.6$, strong agreement [21].

Since there is no gold standard for the determination of LTBI, the analysis was focussed on estimating the strength of association between the degree of exposure, i.e. the proximity between contact persons and the source case. Logistical regression procedures were used to estimate results of odds ratios (ORs) of each test in comparisons between the groups of close and of occasional contacts. Beside proximity of exposure (stratification), variables included age, origin of birth (East or West Germany), ELISPOT and TST result and history of BCG vaccination. All tests were performed as two-sided tests.

\section{RESULTS}

A total of $71(19.2 \%)$ of the 369 contacts developed an induration $\geqslant 5 \mathrm{~mm}$ at the TST site: 19 of the 36 close contacts $(52.8 \%)$ and 52 out of the 333 nonclose contacts $(15.6 \%$; $\mathrm{p}<0.001$ ). According to the current German guidelines [19], this size was taken as a cut-off for positivity in the test. The patients with positive TST results had a mean age \pm SD of $25.9 \pm 11.0$ yrs and were significantly younger than those with negative TST $(29.1 \pm 11.5, \mathrm{p}<0.05)$ and had a similar sex distribution, but differed in respect of origin. In total, 20 out of $74(27.0 \%)$ persons born in the former East Germany had a positive TST result, but only 46 out of the $287(16.0 \%, \mathrm{p}<0.001)$ of those born in the former West Germany did so. Sixty-six out of $74(89.2 \%)$ persons born in East Germany had been previously BCG vaccinated compared with 88 out of the 287 $(30.7 \%)$ born in West Germany $(\mathrm{p}<0.001)$.

One of the close contacts, a 39-yr-old male instructor, developed tuberculous pleuritis in spring 2004 (diagnosis on April 13, 2005). DNA fingerprint results had become available, and recent transmission due to the index case could be confirmed (fig. 2). No other contact developed active TB after contact tracing. In an ongoing population-based epidemiological study in the metropolis of Hamburg, Germany, $\sim 60 \mathrm{~km}$ away from the town of Eutin, isolates from 1,205 patients were investigated by molecular strain typing using IS6110 DNA fingerprint and spoligotyping within the study period 19972004. Results of contact tracing and additional patient interviews have been used for further epidemiological analyses. Among all patients, $\sim 40 \%$ were grouped into clusters based on identical fingerprint patterns of the respective isolates. The size of the restriction fragment length polymorphism clusters ranged from two to 45 isolates; however, the majority of clusters comprise just two isolates $[22,23]$. The particular strain 
involved in the contact investigation in Eutin has caused an outbreak comprising five patients in the study period.

The mean induration size \pm SD of the TST was $13.8 \pm 6.7 \mathrm{~mm}$ in the BCG-vaccinated TST-positives and $16.2 \pm 8.0 \mathrm{~mm}$ in the unvaccinated contacts, but this difference was not statistically significant.

In total, 83 contacts underwent ELISPOT testing (fig. 3; table 1); 56 of the 71 TST-positive contacts $(78.9 \%)$, and 27 out of $298(9.1 \%)$ TST-negative contacts were chosen at random. Fifteen TST-positive contacts declined the offer of ELISPOT testing. In total, agreement between TST and ELISPOT was low $(\kappa=0.16,95 \%$ confidence interval (CI) 0.1 $0.25 ; \mathrm{p}=0.006)$, with concordant results in only 40 out of 83 $(48.2 \%)$, i.e. 13 out of 56 contacts $(23.2 \%)$ with positive TST results (eight out of the 19 TST-positive close contacts $(42.1 \%$ ) and five out of the 37 occasional contacts $(13.5 \%)$ ), but 27 out of $27(100 \%)$ students with negative TST results. Among the nonvaccinated individuals, ELISPOT and TST results were in good agreement $(\kappa=0.5,95 \%$ CI $0.4-0.6 ; p=0.001)$. All of the 18 nonvaccinated contacts who had negative TST results were also ELISPOT negative. Nine out of the 18 who were TSTpositive $(50 \%)$ were also ELISPOT positive

There was no correlation between BCG vaccination and ELISPOT result $(\kappa=0.04)$.

Of all contacts, 158 out of $369(42.8 \%)$ had never received BCG vaccination. Of these, $44(27.8 \%)$ gave a positive TST result. However, of the 211 contacts who had not received BCG vaccination, only $27(12.8 \%)$ gave a positive result $(\mathrm{p}<0.0001)$.

In contrast to this, only four of the 38 TST-positive contacts with previous BCG vaccination submitted to ELISPOT testing gave a positive ELISPOT result $(10.5 \%)$, while nine out of 18 nonvaccinated TST-positive contacts $(50 \%)$ were positive $(\mathrm{p}<0.01)$.

Since the numbers of contacts who underwent TST testing and ESAT-6 testing differed markedly, a logistical regression analysis was used to estimate the odds ratio (OR) between degree of exposure and TST or ELISPOT response, respectively. The 83 contacts for whom an ESAT-6 result was available were included (table 2). Compared with the group of individuals with occasional contact to the index case, the risk of having a positive ELISPOT test was found to be 29.2 times higher (95\% CI 3.5-245.0; $\mathrm{p}=0.002)$, and the risk of having a positive TST 19.7 times higher (95\% CI 2.0-190.2; $\mathrm{p}=0.01$ ) when individuals had close contact to the index case. However, in contrast to ESAT-6 testing (no significant OR) this result was strongly confounded by BCG vaccination (OR 4.8 (95\% CI 1.3-18.0; $\mathrm{p}=0.02)$; table 3).

Following the current guidelines, all individuals with a positive TST (induration $>5 \mathrm{~mm}$ ) were offered 9 months of isoniazid prophylaxis.

\section{DISCUSSION}

In this contact study of a TB outbreak in a police academy in Germany, the degree of exposure to the index case was more closely correlated with the detection of MTB-specific T-cells from PBMCs in an ELISPOT assay than with a positive TST reaction. This finding was independent of age, sex and BCG vaccination status. In a low incidence country for TB with a substantial proportion of individuals who have been vaccinated with BCG in the past, the ELISPOT allowed better discrimination between true infection and cross-reactivity, and can thus circumvent the unpredictable influence of BCG on the

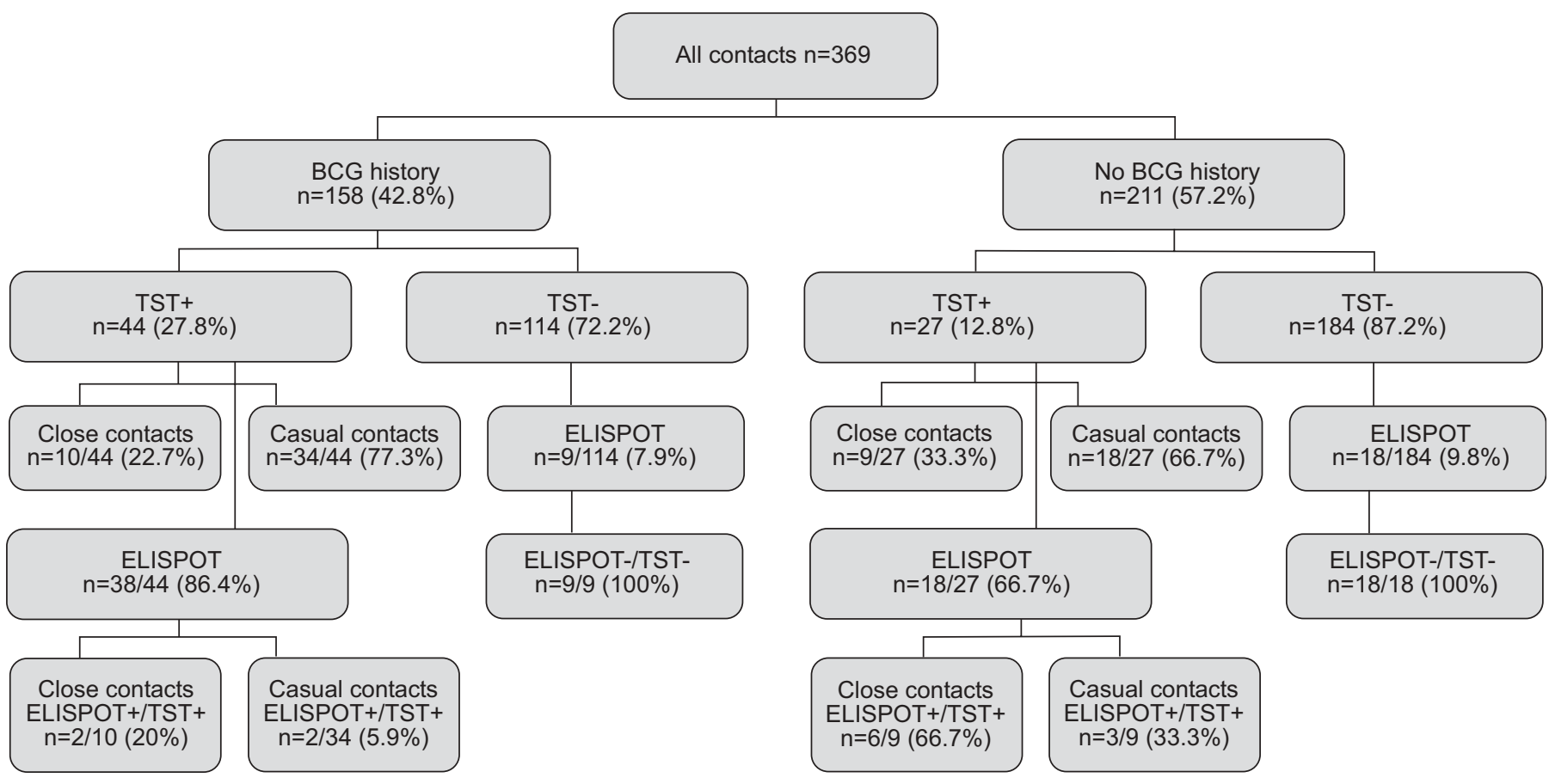

FIGURE 3. Distribution of test results according to the history of bacille Calmette-Guérin (BCG) vaccination. TST: tuberculin skin test; ELISPOT: enzyme-linked immunospot. 
TABLE 1 Results of Mycobacterium tuberculosis-specific interferon- $\gamma$ release assay (ELISPOT) among tuberculin skin test (TST)positive contacts

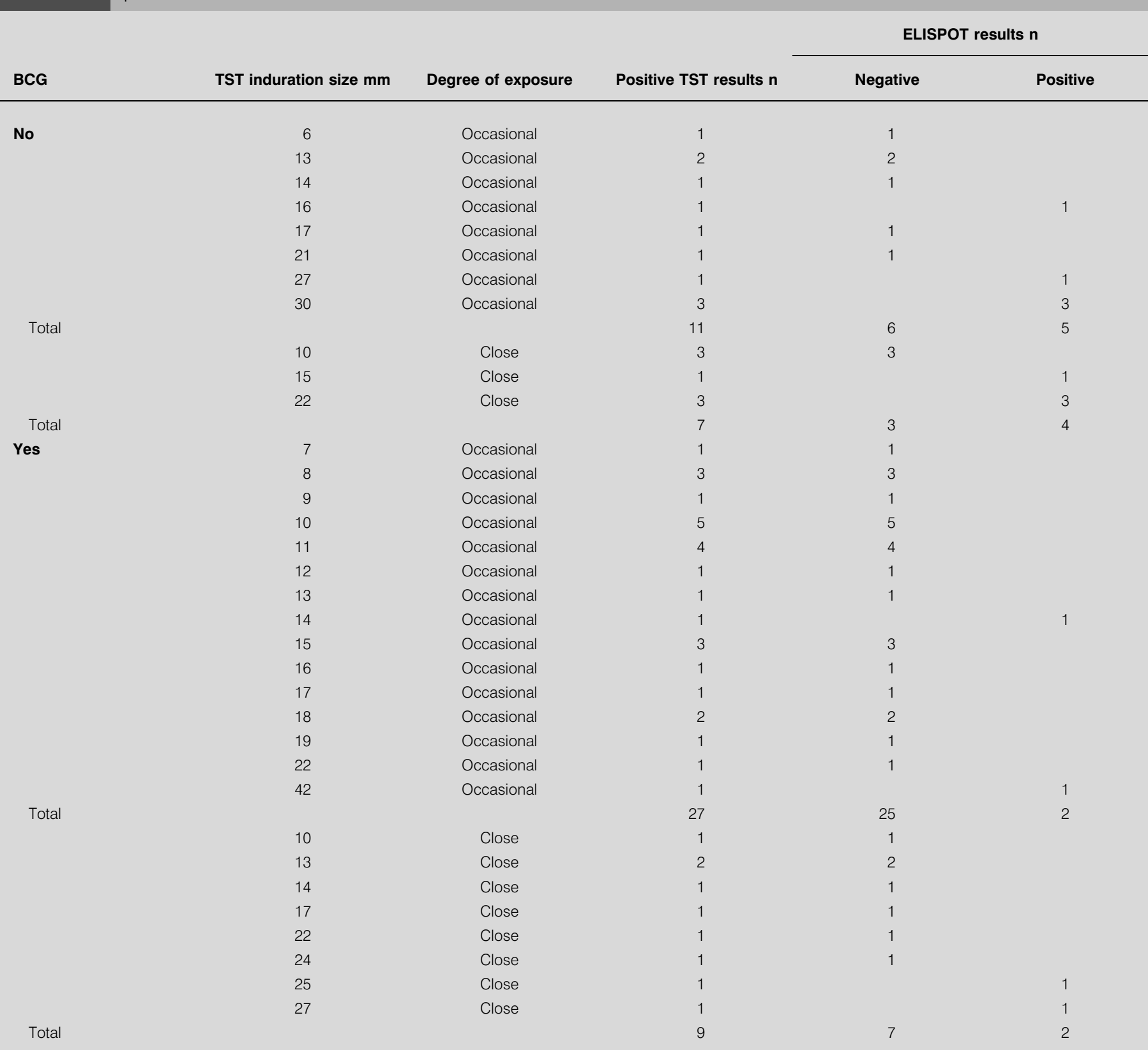

ELISPOT: enzyme-linked immunospot; BCG: bacille Calmette-Guérin.

TST. However, this study was conducted in a healthy, young population, which does not allow the usefulness of this test to be evaluated in an older population with chronic underlying diseases.

Of the 369 contact persons investigated, $71(19.2 \%)$ were TSTpositive. Although the TST is still regarded as the gold standard for the diagnosis of LTBI, TST only offers an indirect diagnosis of LTBI and can produce a substantial number of false-positive test results [13]. Nevertheless, official guidelines [24] require that a positive test result should be followed by treatment. However, this study has revealed two crucial points that affect a decision to start chemotherapy for presumed LTBI on the basis of a positive TST. First, in absolute numbers, there were more TST-positive persons among the occasional than the close contacts, while relatively speaking there were more than twice as many TST-positive persons among those contacts who had previously been vaccinated (44 out of $158(27.9 \%)$ versus 27 out of $211(12.8 \%))$, indicating the possibility of a false positive reaction due to cross-reactivity with $M$. bovis BCG.

The present study demonstrates a poor correlation between the results of TST and those of the ELISPOT assay among 56 TSTpositive contacts when the induration cut-off for the TST was 


\begin{tabular}{|c|c|c|c|c|}
\hline \multirow{2}{*}{$\begin{array}{l}\text { TABLE } 2 \\
\text { Risk factor }\end{array}$} & \multicolumn{4}{|c|}{$\begin{array}{l}\text { Results of multiple logistical regression: odds } \\
\text { ratio for a positive early secretory antigenic target } \\
6 \text { result in a comparison between the groups of } \\
\text { close and occasional contacts }\end{array}$} \\
\hline & & Odds ratio & $95 \% \mathrm{Cl}$ & p-value \\
\hline \multicolumn{2}{|c|}{$\begin{array}{l}\text { Stratification (close versus } \\
\text { occasional contacts) }\end{array}$} & 29.2 & $3.5-245.0$ & 0.002 \\
\hline \multicolumn{2}{|c|}{ Age yrs } & 1.1 & $0.99-1.2$ & $0.6^{\#}$ \\
\hline \multicolumn{2}{|c|}{ Origin (East/West Germany) } & 2.2 & $0.56-8.5$ & $0.3^{\#}$ \\
\hline \multicolumn{2}{|c|}{ BCG vaccination } & 0.31 & $0.05-1.9$ & $0.2^{\#}$ \\
\hline
\end{tabular}

Cl: confidence interval; BCG: bacille Calmette-Guérin. ${ }^{*}$ : nonsignificant.

\begin{tabular}{|c|c|c|c|c|}
\hline \multirow{2}{*}{$\begin{array}{l}\text { TABLE } 3 \\
\text { Risk factor }\end{array}$} & \multicolumn{4}{|c|}{$\begin{array}{l}\text { Odds ratio for a positive tuberculin skin test result } \\
\text { with a cut-off of } 5 \mathrm{~mm}\end{array}$} \\
\hline & & Odds ratio & $95 \% \mathrm{Cl}$ & $\mathrm{p}$-value \\
\hline \multicolumn{2}{|c|}{$\begin{array}{l}\text { Stratification (close versus } \\
\text { occasional contacts) }\end{array}$} & 19.7 & $2.0-190.2$ & 0.01 \\
\hline \multicolumn{2}{|c|}{ Age yrs } & 0.98 & $0.95-1.0$ & $0.9^{\#}$ \\
\hline \multicolumn{2}{|c|}{ Origin (East/West Germany) } & 1.8 & $0.64-5.3$ & $0.3^{\#}$ \\
\hline \multicolumn{2}{|c|}{ BCG vaccination } & 4.8 & $1.3-18.0$ & 0.02 \\
\hline
\end{tabular}

set to a $5 \mathrm{~mm}$ diameter. This remarkable discrepancy could not be explained by false negative IFN- $\gamma$ assay results, because the control group showed an excellent concordance $(\kappa=1.0)$. Moreover, it was surprising that $>80 \%$ of the contacts who had agreed to ELISPOT testing failed to confirm positive TST results. This indicates three possibilities. The first is that ELISPOT is not sensitive enough to confirm a true LTBI. The second is that the cut-off of TST representing a positive result is too low because of an overestimation of the true degree of exposure of a contact and must therefore be raised. The third is that, as in the case of BCG vaccination, there are crossreactivities with other mycobacteria whose infection prevalence cannot be determined, even by laboratory investigations because of the multitude of species that may be present.

Most of the contact individuals in this study who grew up in former East Germany had received BCG vaccination at birth and again, if a TST control showed re-conversion to negative, during early adolescence. However, BCG vaccination was only performed sporadically in West Germany. The results of both the TST- and MTB-specific ELISPOT were sensitive to the degree of contact to the infectious source case. However, owing to the confounding by BCG vaccination, detection of MTBspecific T-cells in the peripheral blood appears to be the better method when a considerable number of contacts have previously been BCG vaccinated. These findings are in agreement with studies in children $[13,19]$ where TST was more likely to be positive in BCG-vaccinated than in nonvaccinated children, whereas INF- $\gamma$ production by MTBspecific T-cells was not associated with BCG vaccination status.
The influence of prior BCG vaccination on the TST- and MTBspecific IFN- $\gamma$ release assays in the detection of LTBI has also been very recently investigated in adults $[19,18,25]$. In these studies, and in agreement with the findings of the present study, only in BCG-unvaccinated individuals were positive MTB-specific IFN- $\gamma$ release assays highly positively correlated with positive TST results. BCG vaccination was a confounder of the TST but not of the IFN- $\gamma$ release assays [13, 18, 25]. Recently a MTB-specific ELISPOT was compared with the TST in contacts of patients with TB in the USA. Among 209 nonBCG-vaccinated persons, frequencies of positive TST and ELISPOT results were almost identical. Even in non-BCGvaccinated persons, ELISPOT tended to be more closely related to the degree of exposure with the index case than the TST [26]

The present study was not designed to investigate the effect of BCG vaccination on MTB-transmission in adults; however, the frequency of a positive MTB-specific ELISPOT was lower in individuals who received BCG vaccination compared with BCG-unvaccinated individuals. This difference could be due to a protective effect of BCG vaccination on MTB infection, as was recently suggested [27]. The strain involved in this outbreak had a normal level of virulence compared with other strains in this low incidence area. Nevertheless, only 13 out of 369 contacts could be confirmed by ELISPOT to have LTBI and only one of these $13(7.7 \%)$ developed active TB within 2 yrs of follow-up. This is a slightly lower rate of secondary cases compared with other outbreaks (e.g. in [19], where nine secondary cases out of 97 ELISPOT-confirmed LTBI cases $(9.2 \%)$ occurred). Secondary cases of TB did not occur among 32 contacts with LTBI in a study in Denmark [13], where the incidence of TB is also low. Of note, prior BCG vaccination did not protect against MTB infection in that study.

The current German guidelines [20] distinguish between close and occasional contacts, but do not distinguish between different cut-off sizes in the evaluation of the TST reaction. However, when the statistical likelihood that a true MTB infection has occurred is limited by a short exposure time, the possibility that a small TST induration represents a false positive reaction increases. Thus, the present authors considered the possibility that adopting a higher cut-off point for positivity in the TST might result in higher levels of agreement between the two tests. Following the current guidelines of the American Thoracic Society (ATS) [28], the cut-off was raised to the next recommended diameter, i.e. $\geqslant 10 \mathrm{~mm}$. This is the cutoff diameter recommended for persons who are at increased risk, but who are not close contacts. This caused an increase in the $\kappa$ value to 0.29 overall and, for the nonvaccinated contacts, to 0.61 , representing strong agreement between both tests. In the logistical regression analysis, the confounding by BCG vanished and the OR for a positive TST finding between the two exposure groups increased to 25.9 (95\% CI 2.8-238.1; table 4).

The fact that increasing the cut-off to $10 \mathrm{~mm}$ for occasional contacts also resulted in a strong agreement between the results from TST and those from ELISPOT in the nonvaccinated population seems to support both the influence of BCG as a confounder and the part played by the actual duration of exposure to the source. This corresponds to the results of other studies, in which a strong agreement between TST and 


\begin{tabular}{|c|c|c|c|c|}
\hline \multirow{2}{*}{$\begin{array}{l}\text { TABLE } 4 \\
\text { Risk factor }\end{array}$} & \multicolumn{4}{|c|}{$\begin{array}{l}\text { Odds ratio for a positive tuberculin skin test result } \\
\text { with a cut-off of } 5 \mathrm{~mm} \text { in close and of } 10 \mathrm{~mm} \text { in } \\
\text { occasional contacts }\end{array}$} \\
\hline & & Odds ratio & $95 \% \mathrm{Cl}$ & p-value \\
\hline \multicolumn{2}{|c|}{$\begin{array}{l}\text { Stratification (close versus } \\
\text { occasional contacts) }\end{array}$} & 25.9 & $2.8-238.1$ & 0.004 \\
\hline \multicolumn{2}{|c|}{ Age yrs } & 0.99 & $0.95-1.1$ & $0.89^{\#}$ \\
\hline \multicolumn{2}{|c|}{ Origin (East/West Germany) } & 0.98 & $0.4-2.4$ & $0.96^{\#}$ \\
\hline \multicolumn{2}{|c|}{$B C G$ vaccination } & 3.5 & $0.98-12.8$ & $0.054^{\#}$ \\
\hline
\end{tabular}

ELISPOT could be seen when a diameter of $10 \mathrm{~mm}$ was used as cut-off from the start [29]. Thus, when the TST is performed for TB contact tracing, a TST with a cut-off of $10 \mathrm{~mm}$, rather than $5 \mathrm{~mm}$, as stated in the ATS/Centers for Disease Control and Prevention and current German Guidelines, should be regarded as positive in BCG-vaccinated individuals and/or occasional contacts. However, even in individuals with no history of BCG vaccination and a TST $>10 \mathrm{~mm}$, a MTB-specific IFN- $\gamma$ release assay may be a better indicator for the risk of LTBI than the TST. For the decision to initiate chemotherapy against LTBI, a TST $>10 \mathrm{~mm}$ should be confirmed by a consecutive MTB-specific IFN- $\gamma$ release that further minimises the number of contacts falsely classified to be MTB-infected.

Blood testing for an IFN- $\gamma$ production by MTB-specific T-cells, either by ELISA or by ELISPOT, have been showing very promising results for the diagnosis of active and latent TB in recent years [11, 16, 18, 30-38]. Tests using MTB-specific antigens, such as ESAT-6 and the cultured filtrated protein-10 out of the region of differences (RD)- 1 in the MTB genome, have a better specificity than the test with PPD antigen $[28,35$, 36,39 ]. The RD-1 is absent in most nontuberculous mycobacteria (with the exception of M. marinum, M. szulgai and $M$. kansasii), and thus false positive results are less likely to be obtained than with the PPD-based TST. The sensitivity of these assays can be increased by using a combination of two antigens, a limitation in the present study. However, specificity of these assays is close to $100 \%$, even when only ESAT- 6 antigen is used [11, 15, 36]. At present it is unclear whether test results with ELISA and ELISPOT are equivalent, and studies comparing the two tests concurrently with the TST are needed.

In conclusion, the data presented here suggest that an Mycobacterium tuberculosis-specific enzyme-linked immunospot is superior to the tuberculin skin test in detecting latent tuberculosis infection and is the test of choice when considering the possibility of administering chemotherapy to persons who have earlier received bacillus Calmette-Guérin vaccination and/or whose contact status is unclear. For occasional and bacillus Calmette-Guérin vaccinated contacts, the tuberculin skin test cut-off should be raised from 5 to $10 \mathrm{~mm}$ in order to minimise the number of false positive results. This study showed that the knowledge of the bacillus Calmette-Guérin vaccination status is important in the diagnosis of latent tuberculosis infection.

\section{ACKNOWLEDGEMENTS}

The authors would like to thank R. Reichenbach and J. Schenck for technical assistance at the Research Center Borstel and the staff of the Medical Service at the Police Academy SchleswigHolstein for their assistance.

\section{REFERENCES}

1 Ramachandran R, Paramasivan $\mathrm{CN}$. What is new in the diagnosis of tuberculosis? Ind J Tub 2003; 50: 133-150.

2 Global tuberculosis control: surveillance, planning, financing. WHO report 2005. Geneva, World Health Organization (WHO/HTM/TB/2005.349). www.who.int/ $\mathrm{tb} /$ publications/global_report/en/index.html. Date last accessed: March 31, 2006.

3 Bradford WZ, Koehler J, Hiyam EH, et al. Dissemination of Mycobacterium tuberculosis across the San Francisco Bay Area. J Infect Dis 1988; 177: 1104-1107.

4 Gutierrez MC, Vincent V, Aubert D, et al. Molecular fingerprinting of Mycobacterium tuberculosis and risk factors for tuberculosis transmission in Paris, France, and surrounding area. J Clin Microbiol 1998; 36: 486-492.

5 Van Soolingen D, Borgdorff MW, de Haas PE, et al. Molecular epidemiology of tuberculosis in the Netherlands: a nationwide study from 1993 through 1997. J Infect Dis 1999; 3: 726-736.

6 Alland D, Kalkut GE, Moss AR, et al. Transmission of tuberculosis in New York City. An analysis by DNA fingerprinting and conventional epidemiological methods. N Engl J Med 1994; 330: 1710-1716.

7 Small PM, Hopewell PC, Singh SP, et al. The epidemiology of tuberculosis in San Francisco. A population-based study using conventional and molecular methods. N Engl J Med 1994; 330: 1703-1709.

8 Diel R, Nienhaus A, Schaberg T. Cost-effectiveness of isoniazid chemoprevention in close contacts. Eur Respir J 2005; 26: 465-473.

9 Andersen P, Munk ME, Pollock JM, Doherty TM. Specific immune-based diagnosis of tuberculosis. Lancet 2000; 356: 1099-1104.

10 Wilkinson KA, Wilkinson RJ, Pathan A, et al. Ex vivo characterization of early secretory antigen target 6-specific $\mathrm{T}$ cells at sites of active disease in pleural tuberculosis. Clin Infect Dis 2005; 40: 184-187.

11 Johnson PD, Stuart RL, Grayson ML, et al. Tuberculinpurified protein derivative-, MPT-64-, and ESAT-6-stimulated gamma interferon responses in medical students before and after Mycobacterium bovis BCG vaccination and inpatients with tuberculosis. Clin Diagn Lab Immunol 1999; 6: 934-937.

12 Brock I, Munk ME, Kok-Jensen A, Andersen P. Performance of whole blood IFN-gamma test for tuberculosis diagnosis based on PPD or the specific antigens ESAT-6 and CFP-10. Int J Tuberc Lung Dis 2001; 5: 462-467.

13 Brock I, Weldingh K, Lillebaek T, Follmann F, Andersen P. Comparison of tuberculin skin test and new specific blood test in tuberculosis contacts. Am J Respir Crit Care Med 2004; 170: 65-69.

14 Ravn P, Munk ME, Andersen AB, et al. Reactivation of tuberculosis during immunosuppressive treatment in a 
patient with a positive QuantiFERON-RD1 test. Scand J Infect Dis 2004; 36: 499-501.

15 Lalvani A, Pathan AA, McShane H, et al. Rapid detection of Mycobacterium tuberculosis infection by enumeration of antigen-specific T cells. Am J Respir Crit Care Med 2001; 163: 824-828.

16 Lalvani A, Nagvenkar P, Udwadia Z, et al. Enumeration of $\mathrm{T}$ cells specific for RD1-encoded antigens suggests a high prevalence of latent Mycobacterium tuberculosis infection in healthy urban Indians. J Infect Dis 2001; 183: 469-477.

17 Whalen CC. Diagnosis of latent tuberculosis infection. JAMA 2005; 293: 2785-2787.

18 Kang YA, Lee HW, Yoon HI, et al. Discrepancy between the tuberculin skin test and the whole-blood interferon gamma assay for the diagnosis of latent tuberculosis infection in an intermediate tuberculosis-burden country. JAMA 2005; 293: 2756-2761.

19 Ewer K, Deeks J, Alvarez L, et al. Comparison of T-cellbased assay with tuberculin skin test for diagnosis of Mycobacterium tuberculosis infection in a school tuberculosis outbreak. Lancet 2003; 361: 1168-1173.

20 Deutsches Zentralkomitee zur Bekämpfung der Tuberkulose. [Central Committee for control of tuberculosis.] Richtlinien für die Umgebungsuntersuchungen bei Tuberkulose. [Guidelines for environmental contact tracing in tuberculosis.] Gesundheitswesen 1996; 58: 657-665.

21 Sachs L. Applied statistics. Application of Statistical Methods. 10th Edn. Berlin, Springer, 2002.

22 Diel R, Schneider S, Meywald-Walter K, Ruf C-M, RüschGerdes S, Niemann S. Epidemiology of tuberculosis in Hamburg, Germany: long-term population-based analysis in applying classical and molecular epidemiological techniques. J Clin Microbiol 2002; 40: 532-539.

23 Diel R, Nienhaus A, Seidler A, Niemann S. Occupational risk of tuberculosis transmission in Hamburg, Germany - a long-term population-based molecular-epidemiological survey 1997-2002. Respir Res 2004; 6: 35.

24 Schaberg T, Hauer B, Loddenkemper R, et al. Latent tuberculosis infection: recommendations for preventive therapy in adults in Germany. Pneumologie 2004; 58: 92-102.

25 Zellweger JP, Zellweger A, Ansermet S, de Senarclens B, Wrighton-Smith P. Contact tracing using a new T-cellbased test: better correlation with tuberculosis exposure than the tuberculin skin test. Int J Tuberc Lung Dis 2005; 9: 1242-1247.

26 Shams H, Weis SE, Klucar P, et al. Enzyme-linked immunospot and tuberculin skin testing to detect latent tuberculosis infection. Am J Respir Crit Care Med 2005; 172: 1161-1168.
27 Soysal A, Millington KA, Bakir M, et al. Effect of BCG vaccination on risk of Mycobacterium tuberculosis infection in children with household tuberculosis contact: a prospective community-based study. Lancet 2005; 366: 1443-1451.

28 American Thoracic Society. Targeted tuberculin testing and treatment of latent tuberculosis infection. Am J Respir Crit Care Med 2000; 161: S221-S247.

29 Hill PC, Fox A, Jeffreis DJ, et al. Quantitative T cell assay reflects infectious load of Mycobacterium tuberculosis in an endemic case contact model. Clin Infect Dis 2005; 490: 273-278.

30 Lalvani A, Pathan AA, Durkan H, et al. Enhanced contact tracing and spatial tracking of Mycobacterium tuberculosis infection by enumeration of antigen-specific T cells. Lancet 2001; 357: 2017-2021.

31 Dheda K, Rook G, Zumla A. Peripheral T cell interferongamma responses and latent tuberculosis. Am J Respir Crit Care Med 2004; 170: 97-98.

32 Dheda K, Udwadia ZF, Huggett JF, Johnson MA, Rook GA. Utility of the antigen-specific interferon-gamma assay for the management of tuberculosis. Curr Opin Pulm Med 2005; 11: 195-202.

33 Chapman AL, Munkanta M, Wilkinson KA, et al. Rapid detection of active and latent tuberculosis infection in HIVpositive individuals by enumeration of Mycobacterium tuberculosis-specific T cells. AIDS 2002; 16: 2285-2293.

34 Mori T, Sakatani M, Yamagishi F, et al. Specific detection of tuberculosis infection: an interferon-gamma-based assay using new antigens. Am J Respir Crit Care Med 2004; 170: 59-64.

35 Ravn $\mathrm{P}$, Munk ME, Andersen $\mathrm{AB}$, et al. Prospective evaluation of a whole-blood test using Mycobacterium tuberculosis-specific antigens ESAT-6 and CFP-10 for diagnosis of active tuberculosis. Clin Diagn Lab Immunol 2005; 12: 491-496.

36 Pathan AA, Wilkinson KA, Klenerman P, et al. Direct ex vivo analysis of antigen-specific IFN-gamma-secreting CD4 $\mathrm{T}$ cells in Mycobacterium tuberculosis-infected individuals: associations with clinical disease state and effect of treatment. J Immunol 2001; 167: 5217-5225.

37 Pai M, Gokhale K, Joshi R, et al. Mycobacterium tuberculosis infection in health care workers in rural India: comparison of a whole-blood interferon gamma assay with tuberculin skin testing. JAMA 2005; 293: 2746-2755.

38 Pai M, Riley LW, Colford JM Jr. Interferon-gamma assays in the immunodiagnosis of tuberculosis: a systematic review. Lancet Infect Dis 2004; 4: 761-776.

39 Rothel JS, Andersen P. Diagnosis of latent Mycobacterium tuberculosis infection: is the demise of the Mantoux test imminent? Expert Rev Anti Infect Ther 2005; 3: 981-993. 